\title{
Simulation of the Oxidation and Combustion of Mixed Diesel- Biodiesel Fuel
}

\author{
Yuswan Muharam ${ }^{1, *}$, Danny Leonardi ${ }^{1}$, and Alisya P Ramadhania ${ }^{1}$ \\ ${ }^{1}$ Department of Chemical Engineering, Faculty of Engineering, University of Indonesia, UI Campus, Depok 16424, Indonesia
}

\begin{abstract}
A comparative simulation-based research has been set up to obtain valid kinetic models of the oxidation and combustion of biodiesel surrogate and diesel surrogate, as well as mixed diesel-biodiesel surrogates which is used to predict their ignition delay times (IDT). The research consists of the development of the detailed kinetic models of the oxidation and combustion of biodiesel surrogate and diesel surrogate, the validation of the two models with the corresponding experimental IDT data, the merging and the validation of the two models for mixed diesel-biodiesel surrogates. The biodiesel surrogate kinetic model was validated with the experimental IDT data of methyl 9-decenoate at 20 atm and three equivalence ratios. The diesel surrogate kinetic model was validated with the experimental IDT data of n-hexadecane at the pressure ranging from $2 \mathrm{~atm}$ to $5 \mathrm{~atm}$ and the equivalence ratio of 1.0. The diesel-biodiesel surrogate kinetic model was validated with the experimental IDT data of real diesel-biodiesel fuels for four compositions and at $1.18 \mathrm{~atm}$. The validation results of all models show that the models and the experiments are in good agreement.
\end{abstract}

\footnotetext{
${ }^{*}$ Corresponding author: muharam@che.ui.ac.id
} 


\section{Introduction}

People's needs for fuel keep rising for the last decades as people's living standards have been raised continuously, along with the growth of industrial development. For the last 30 years, one of the sectors which undergoes continuous increase in fuel consumption is the transportation sector with percentages as high as $30 \%$ of total energy consumption in the world. In this case, almost all fuel consumed for transportation purposes is derived from fossil sources. With the continuous depletion of crude oil reserves each year, some solutions for alternative energy sources have been suggested and verified, one of it being biodiesel. Biodiesel is a type of fuel equivalent to diesel fuel in terms of usage and properties, but derived from natural sources.

Recently, the development of biodiesel has become the central theme of many researches, due to its renewable properties and less $\mathrm{CO}_{2}$ emission throughout its life cycle [1]. But in its real application in daily lives, especially in Indonesia, biodiesel is still used in mixtures with diesel fuel due to some of its different physical properties compared to normal diesel fuel. Biodiesel has higher oxygen content, higher viscosity and lower volatility, which contribute to negative effects towards the diesel engine in which the fuel is used [1]. However, biodiesel has higher cetane number compared to normal diesel fuel leading to shorter ignition delay times (IDT). This is considered as an advantage in terms of diesel engine combustion process.

Ignition delay time is defined as the time lapse which is measured between the start of fuel injection and the start of combustion in a diesel engine. Many factors influence IDT, but it can be generally divided into two; physical factor and chemical factor. The physical factors are fuel atomization process, fuel vaporization, and mixing with air in a combustion chamber. The chemical factors include elementary chemical reactions occurring before and during the combustion [2]. This factor depends on the reaction mechanism of the fuel. The difference of molecular structures of fuels differs the reaction mechanisms resulting in different IDTs. One of the methods to approach this problem is simulation of kinetics of reaction mechanisms of these fuels.

Many studies have been carried out to characterize combustion processes involving biodiesel and diesel fuels. Some of these studies were also intended to find out new surrogate compounds or to examine existing surrogates generating similar combustion characteristics to the fuel without simulating the full composition of the fuel itself. In general, surrogate compounds suitable to fit the combustion characteristics of biodiesel are methyl esters with short to medium-length carbon chain. In case of diesel fuel, the surrogate compounds might involve hydrocarbons with long carbon chain $\left(\mathrm{C}_{12}\right.$ or longer).

Herbinet et al. [3] developed a detailed combustion kinetic model for biodiesel surrogates in the form of medium-length methyl esters with a double-bond, such as methyl 9-decenoate and methyl-5-decenoate. Methyl 9-deceonate is chosen because its double bond is at the same position as the one in methyl oleate and at the same location as the first double bond in methyl linoleate and in methyl linolenate. Westbrook et al. [4] developed a combustion kinetic model to simulate normal hydrocarbons with carbon chains being longer than octane, ranging from $\mathrm{C}_{8}$ to $\mathrm{C}_{16}$. The kinetic model uses reaction routes and classes which is similar to the ones used in the kinetic model of isooctane by Curran et al. [5].

Muharam et al. [6] simulated the IDT of various commercial fuels to find out their octane number. The IDTs of commercial fuels were compared to the ones of the primary reference fuel consisting of $n$-heptane and isooctane.

The objective of the present work is to have valid kinetic models of detailed reaction mechanisms of the oxidation and combustion of a biodiesel surrogate, a diesel surrogate, and a diesel-biodiesel surrogates to predict their IDTs.

\section{Method}

The models in the present work are based on the ones developed by Westbrook et al. [4] for diesel surrogate which is n-hexadecane, and by Herbinet et al. [3] for biodiesel surrogate which is methyl 9-decenoate. Both models have been validated by corresponding researchers. We re-validate both models if there are still IDT differences with the experimental results. The point of this is that when the two models are emerged to give a diesel-biodiesel model, the model can reproduce well the experimental real diesel-biodiesel IDT.

The IDT experimental data for the validation are the ones obtained by Wang et al. [7] for methyl-9-decenoate, and by Haylett et al. [8] for n-hexadecane.

The model for a diesel-biodiesel surrogates is the merging of the biodiesel surrogate model and the diesel surrogate model that have been validated. The IDT experimental data for the validation of diesel-biodiesel model is obtained from Hoang et al. [9] using real dieselbiodiesel fuel.

The validation was performed by changing the values of pre-exponential factors ( $\left.A^{\prime}\right)$ of several reactions giving high sensitivities to IDT. The determination of high sensitivity reactions is carried out through sensitivity analysis.

\section{Model}

In chemistry a reaction mechanism is a step by step sequence of elementary reactions by which overall chemical change occurs [10]. If many elementary reactions exist, the net amount of a reactant consumed or a product produced arise from the addition of the contributions of each elementary reaction. In general, species takes part as a reactant in a reaction and as a product in others. The rates of the production and the consumption are a net result of all elementary reactions.

For a mechanism consisting of $\mathrm{R}$ elementary reactions of S species, which is given by [11] 


$$
\sum_{s=1}^{\mathrm{S}} v_{r s}^{(\mathrm{e})} \mathrm{A}_{s} \stackrel{k_{r}}{\longrightarrow} \sum_{s=1}^{\mathrm{S}} v_{r s}^{(\mathrm{p})} \mathrm{A}_{s}
$$

where $r=1,2, \ldots, \mathrm{R}, v_{r s}^{(\mathrm{e})}$ and $v_{r s}^{(\mathrm{p})}$ indicate the stoichiometric coefficients of reactants and products, respectively, the rate of the formation of a species $i$ is obtained from the addition of the whole rate equations where the species $i$ is involved,

$$
\left(\frac{\partial c_{i}}{\partial t}\right)_{\text {chem }}=\sum_{r=1}^{\mathrm{R}} k_{r}\left(v_{r i}^{(\mathrm{p})}-v_{r i}^{(\mathrm{e})}\right) \prod_{s=1}^{\mathrm{S}} c_{s}^{\nu_{s}^{(\mathrm{e})}}
$$

where $i=1,2, \ldots, \mathrm{S}$.

The rate laws for a reaction mechanism consisting of $\mathrm{R}$ reactions among $\mathrm{S}$ species can be written as a system of first order ordinary differential equations,

$$
\frac{d c_{i}}{d t}=F_{i}\left(c_{1}, \ldots, c_{\mathrm{S}}, k_{1}, \ldots, k_{\mathrm{R}}\right) \quad i=1,2, \ldots, \mathrm{S}
$$

with initial conditions being

$$
c_{i}=c_{i}^{0} \text { at } t=t_{0}
$$

For many elementary reactions, a change in the parameters of the system (the rate coefficients) has nearly no effect on the time-dependent solution. Even if the reaction is included explicitly in the mechanism, it does not need a highly accurate rate coefficient. On the other hand, for a few of the elementary reactions, changes in the rate coefficients have really large effects on the outcome of the system. Accordingly, accurate rate coefficients are demanded. These few important reaction steps are rate-determining steps or rate-limiting steps. The dependence of the solution $c_{i}$ on the parameters $k$ is called sensitivity and sensitivity analysis is a procedure to validate the kinetic model of detailed reaction mechanism.

\section{Results and Discussion}

\subsection{Diesel surrogate}

As mentioned in [4] the diesel surrogate mechanism includes both high temperature and low temperature reaction pathways. The validation of the diesel surrogate model was performed at the temperature range of 1100 $\mathrm{K}-1400 \mathrm{~K}$, the equivalence ratio of 1 , and the pressure of $2 \mathrm{~atm}-5 \mathrm{~atm}$.

The IDTs calculated using the diesel surrogate model is initially much lower than the ones from the experiments. Therefore, the values of pre-exponential factors of reactions with positive sensitivity need to be decreased, and/or of reactions with negative sensitivity need to be increased so that the calculated IDTs slow down and approache the experimental IDTs. The changes were made to the reactions with the highest sensitivity to IDT according to their order. In case of the diesel surrogate, we simply changed the reaction with the highest sensitivity to IDT, i.e. $\mathrm{C}_{3} \mathrm{H}_{5}+\mathrm{HO}_{2} \leftrightarrow \mathrm{C}_{3} \mathrm{H}_{5} \mathrm{O}$ $+\mathrm{OH}$, the best fit was obtained, as shown in Figure 1. 

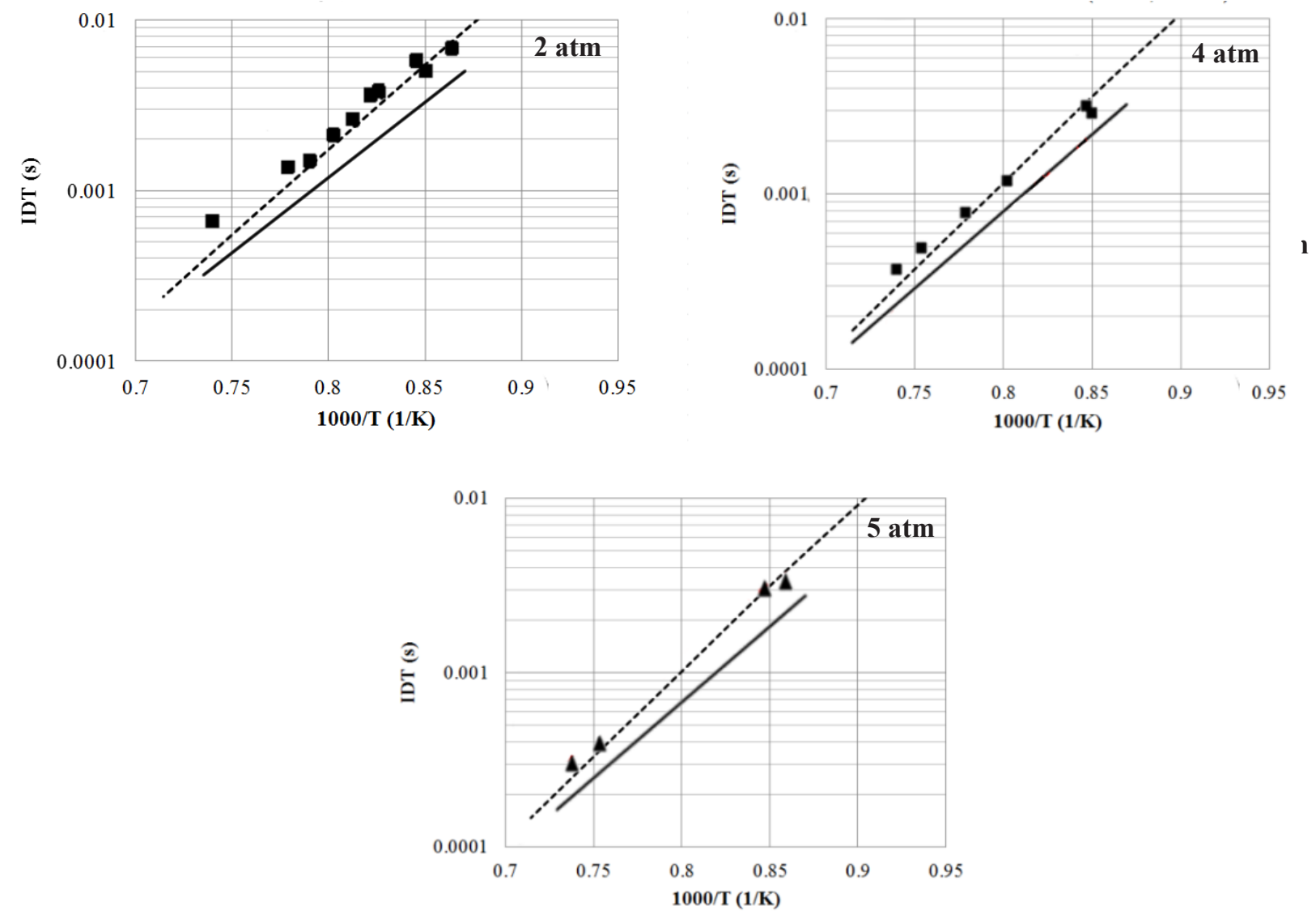

Fig. 1. Comparison of calculated and experimental IDTs of diesel surrogate

( $\boldsymbol{\Lambda}$ and $\mathbf{a r e}$ experimental data, - is simulation by Haylett et al. [8], and - - - is simulation by present work).

From Figure 1, it can be seen that the calculated IDTs are in good agreement with the experimental ones at 2 atm, $4 \mathrm{~atm}$, and $5 \mathrm{~atm}$. At $5 \mathrm{~atm}$ and $1325 \mathrm{~K}$, the calculated IDT is $0.37 \mathrm{~ms}$ and the experimental IDT is $0.38 \mathrm{~ms}$. The calculated and experimental results show that IDTs are faster when the initial temperature and pressure increase. The figure exhibits also the calculation results performed by Haylett et al [8].

\subsection{Biodiesel surrogate}

Validation of the kinetic model of biodiesel surrogate was carried out at three different temperature zones: low, medium and high temperatures, the pressure of $20 \mathrm{~atm}$ and the equivalence ratios of $0.5,1.0$, and 1.5. First the simulation results show the deviation of the calculated IDTs against the experimental ones at low-medium temperature $(700 \mathrm{~K}-900 \mathrm{~K})$. The deviation is negative for all equivalence ratios. Since the deviation values are different among the equivalence ratios, the method of adjusting the kinetic parameters is to find the optimum position between the simulation results and the experimental ones. The optimum position may not precisely match the calculated results with the experimental ones, but at least an approach was carried out such that the simulation results of one of the equivalence ratios may coincide with the experimental results with minimum deviation and good curve shape (the NTC profile at medium temperature remains visible). Therefore, the following optimization steps may be carried out:

1. Low temperature zone: Increasing the values of preexponential factors in reactions with positive sensitivity, and/or decreasing the values of preexponential factors in reactions with negative sensitivity with the emphasis being on the highest sensitivity reactions in the low temperature zone.

2. Medium temperature zone: Performing the same activities with the above, but with advance notice to the IDT simulation results due to the change in the values of pre-exponential factors in the low temperature zone. When the results are good enough, no changes are needed for sensitive reactions in the medium temperature zone. If the adjustment of preexponential factors is necessary, it is done by noting that the reactions with the modified pre-exponential factors in the low temperature zone can not be changed in the medium temperature zone. 


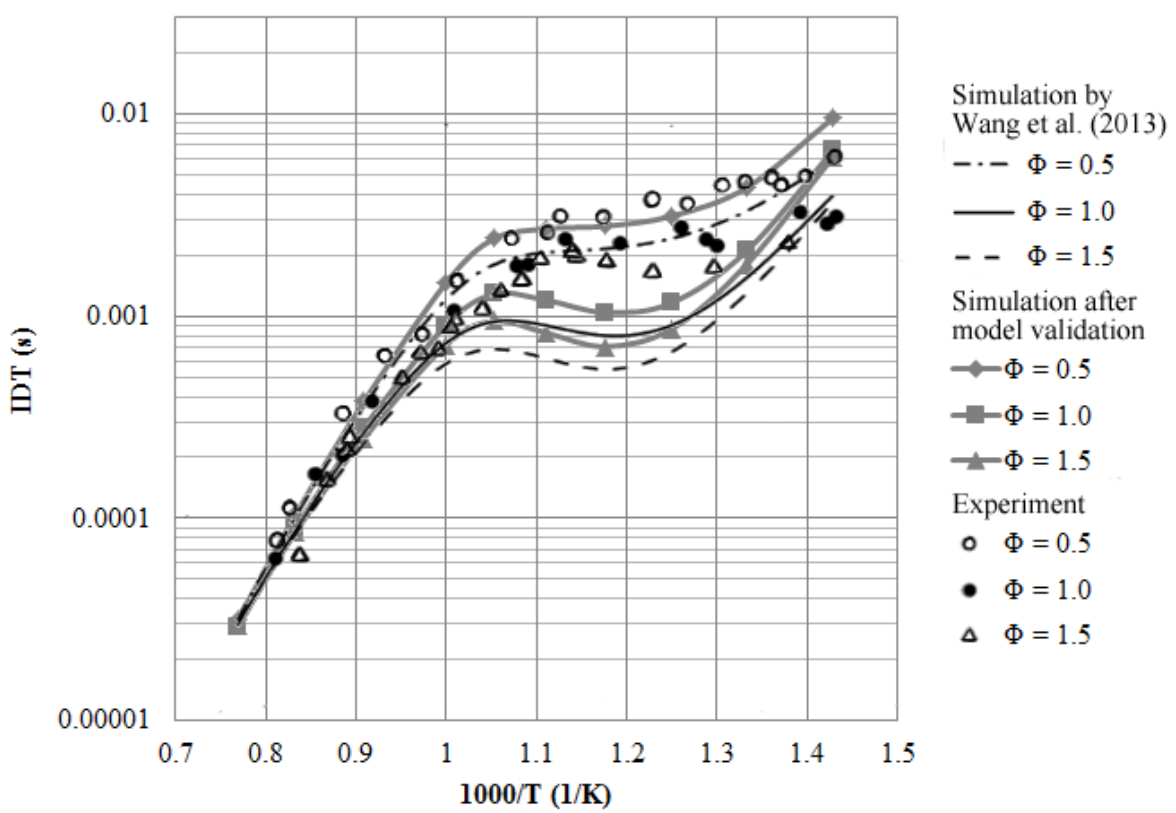

Fig. 2. Comparison between the calculated and experimental IDTs of biodiesel surrogate. (Dots represent experimental data, lines represent simulation results).
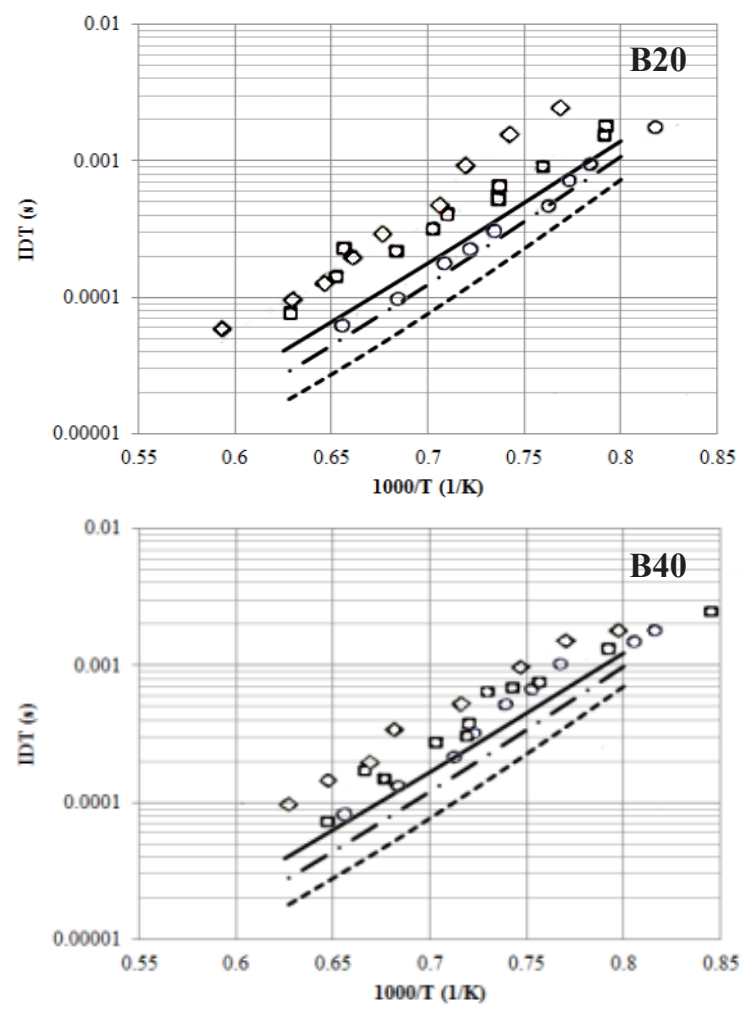
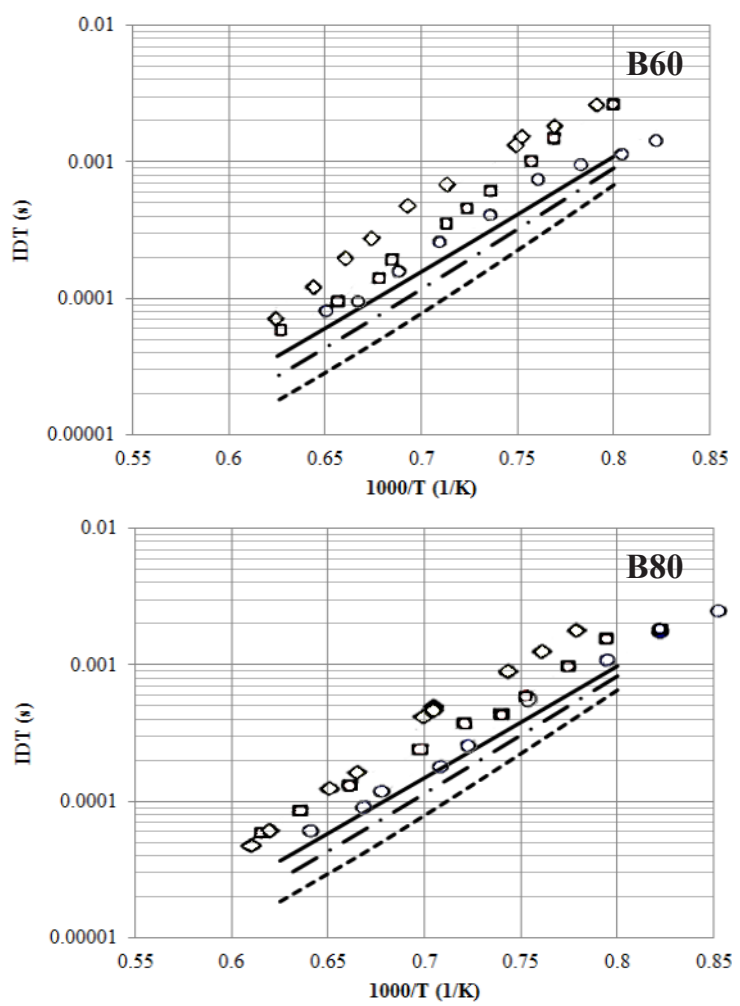

Fig. 3. Comparison between calculated and experimental IDTs of mixed diesel biodiesel fuel before validation $(\circ, \square, \diamond$ experimental data for the equivalence ratios of $0.5,1.0,1.5$; $---,-\cdot,-$ simulation results for the equivalence ratios of $0.5,1.0,1.5)$.

The reactions to which the Arrhenius parameters are changed are: $\mathrm{H}$ atom abstraction from the fuel by oxygen, peroxy $\mathrm{C}_{11} \mathrm{H}_{19} \mathrm{O}_{2}$ radical isomerization, $\mathrm{O}-\mathrm{O}$ scission on peroxy $\mathrm{C}_{11} \mathrm{H}_{19} \mathrm{O}_{2}$ hydroperoxy radical, and decomposition of peroxy $\mathrm{C}_{11} \mathrm{H}_{19} \mathrm{O}_{2}$ oxy radical.
Figure 2 shows the calculated IDTs in this study and the experimental ones. From the figure it can be seen that the calculated IDTs for the equivalence ratio of 0.5 have good agreement with the experimental ones, while those for the equivalence ratios of 1.0 and 1.5 are not entirely agreed, especially in the low-medium temperature range 

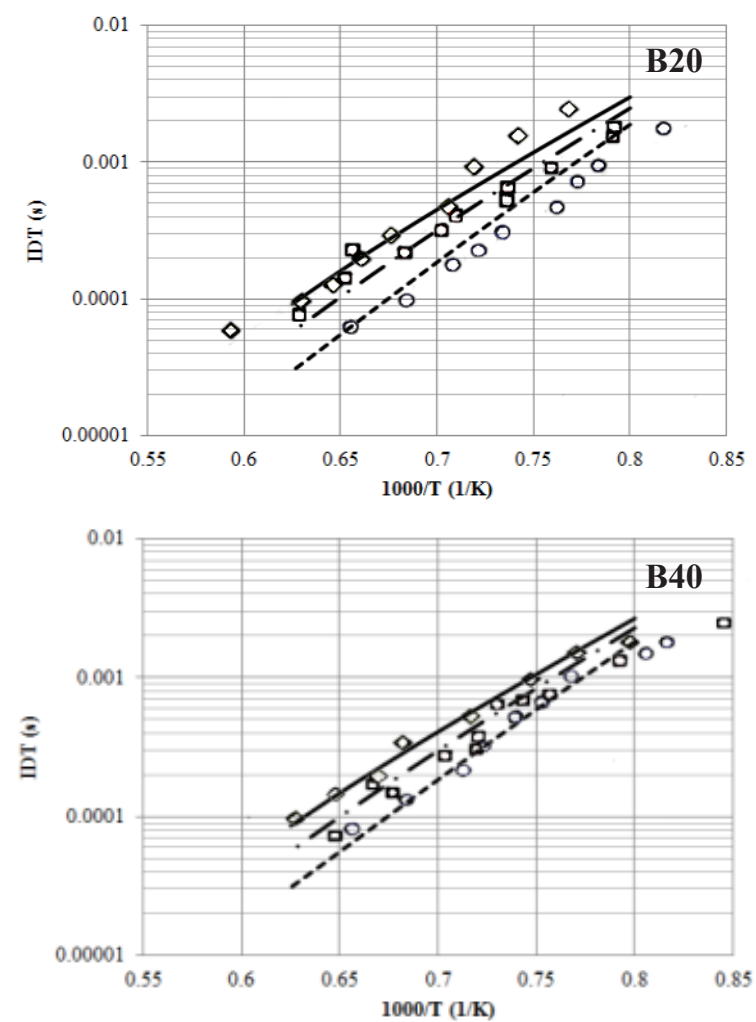
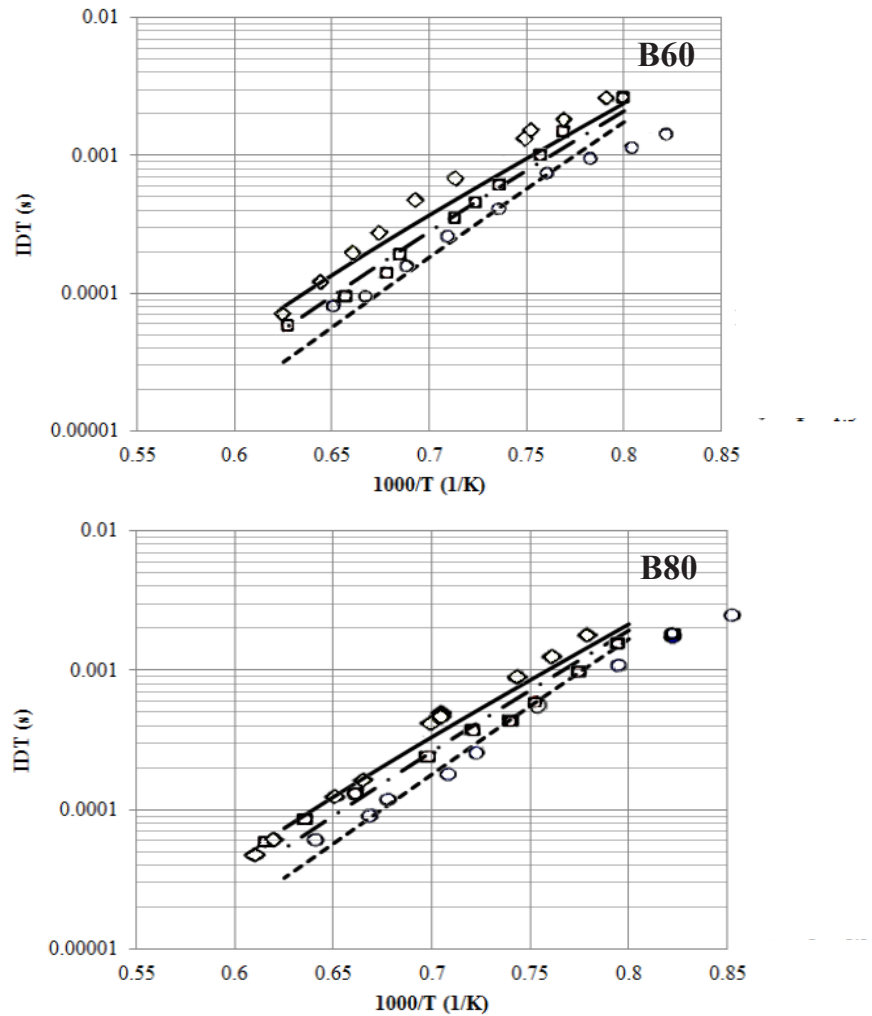

Fig. 4. Comparison between calculated and experimental IDTs of mixed diesel biodiesel fuel after validation $(\circ, \square, \diamond$ experimental data for the equivalence ratios of $0.5,1.0,1.5$;

$---,-\cdot,-$ simulation results for the equivalence ratios of $0.5,1.0,1.5)$.

(750-950 K). When compared with the simulation results by Wang et al. [7], the IDT results in this study have better agreement. At $900 \mathrm{~K}$, the simulation yields the IDT of $0.37 \mathrm{~ms}$, while the experimental data is $0.38 \mathrm{~ms}$.

The calculated and experimental results show the IDT behavior in three temperature zones. In the low and high temperature zones, IDTs are faster with increasing temperature. In the medium temperate zone, the opposite situation occurs where the IDTs slow with increasing temperature.

\subsection{Mixed diesel-biodiesel}

The IDT simulation for the mixed diesel-biodiesel surrogates was carried out in the temperature range of $1200 \mathrm{~K}-1600 \mathrm{~K}$, the pressure of $1.18 \mathrm{~atm}$, and for the fuel composition of B20 (biodiesel 20\%), B40, B60, and B80.

The IDT simulation results are shown in Figure 3. From the figure it is noticed that there is a considerable deviation between the calculated IDTs and the experimental ones. This is because the kinetic model uses diesel and biodiesel surrogates, while the experiment uses real multicomponent diesel-biodiesel fuel.

Since the deviation is negative, it is necessary to adjust the kinetic parameter, i.e. decreasing $A$ ' values in the reactions with positive sensitivity, and/or increasing $A^{\prime}$ values in the reactions with negative sensitivity. With such a high deviation, it is necessary to change the $A^{\prime}$ values of many reactions as follows:

$$
\begin{aligned}
& \mathrm{CH}_{3}+\mathrm{HO}_{2} \leftrightarrow \mathrm{CH}_{3} \mathrm{O}+\mathrm{OH} \\
& \mathrm{C}_{2} \mathrm{H}_{3}+\mathrm{O}_{2} \leftrightarrow \mathrm{CH}_{2} \mathrm{CHO}+\mathrm{O} \\
& \mathrm{C}_{3} \mathrm{H}_{5}+\mathrm{O}_{2} \leftrightarrow \mathrm{C}_{2} \mathrm{H}_{3} \mathrm{CHO}+\mathrm{OH} \\
& \mathrm{C}_{3} \mathrm{H}_{5}+\mathrm{O}_{2} \rightarrow \mathrm{C}_{2} \mathrm{H}_{2}+\mathrm{CH}_{2} \mathrm{O}+\mathrm{OH} \\
& \mathrm{C}_{14} \mathrm{H}_{29}+\mathrm{C}_{2} \mathrm{H}_{5} \leftrightarrow \mathrm{nC}_{16} \mathrm{H}_{34} \\
& \mathrm{C}_{11} \mathrm{H}_{20} \mathrm{O}_{2} \leftrightarrow \mathrm{C}_{8} \mathrm{H}_{15} \mathrm{O}_{2}+\mathrm{C}_{3} \mathrm{H}_{5}
\end{aligned}
$$

Figure 4 shows the calculated and experimental IDTs for mixed diesel-biodiesel fuel. From the figure it can be seen that in general the calculated IDTs after adjustment of some kinetics parameters have good agreement with the experimental IDTs for each composition. The best match is for B40. At $1350 \mathrm{~K}$ and the equivalence ratio 1.0 , the simulated IDT is $0.699 \mathrm{~ms}$ and the experimental one is $0.69 \mathrm{~ms}$.

\section{Conclusions}

The kinetic model of detailed reaction mechanism of the oxidation and combustion of diesel surrogate has been validated with the experimental IDT data at the pressure ranging from $2 \mathrm{~atm}$ to $5 \mathrm{~atm}$ and the equivalence ratio of 1.0. The kinetic model of detailed reaction mechanism of the oxidation and combustion of biodiesel surrogate has been validated with the experimental IDT data at 20 atm and three equivalence ratios. The kinetic model of detailed reaction mechanism of the oxidation and combustion of diesel-biodiesel surrogates has been validated with the experimental IDT data of real biodiesel-diesel fuel for four mixture compositions and at $1.18 \mathrm{~atm}$. In general, the validation results of all 
models show good aggrement between the models and the experiments.

We express our gratitude to the University of Indonesia, which funded this research through the scheme of Hibah Publikasi Internasional Terindeks untuk Tugas Akhir Mahasiswa No 864/UN2.R3.1/HKP.05.00/2017.

\section{References}

1. A.E. Atabani et al., Renew. Sust. Ener. Rev. 16, 2070 (2012)

2. P.A. Lakshminarayanan, Y.V. Aghav, Modelling Diesel Combustion (Springer, 2010)

3. O. Herbinet, W.J. Pitz, C.K. Westbrook, Combust. Flame 157, 893 (2009)

4. C.K. Westbrook, W.J. Pitz, O. Herbinet, H.J. Curran, E.J. Silke, Combust. Flame 156, 181 (2009)

5. H.J. Curran, P. Gaffuri, W.J. Pitz, C.K. Westbrook, Combust. Flame 129, 253 (2002)

6. Y. Muharam, C. Hadiwijaya, Reaktor 14(2), 109 (2012)

7. W. Wang, S. Gowdagiri, M.A. Oehlschlaeger, Ener. Fuels 27, 5527 (2013)

8. D.R. Haylett, D.F. Davidson, R.K. Hanson, Combust. Flame 159, 552 (2011)

9. V.N. Hoang, L.D. Thi, Biosys. Eng. 134, 1 (2015)

10. J. March, Advanced Organic Chemistry: Reactions, Mechanisms, and Structure (Wiley, New York, 1985)

11. M. Nehse, J. Warnatz, C. Chevalier, P. Louessard, H. Melenk, MOLEC: A Program for the Generation of Chemical Reaction Equations, User's Manual (Interdisziplinares Zentrum fur Wissenschaftliches Rechnen Universitaet Heidelberg, Heidelberg, 1996) 